\title{
TWIST-SPUN TORUS KNOTS
}

\author{
C. MCA. GORDON ${ }^{1}$
}

\begin{abstract}
Zeeman has shown that the complement of a twistspun knot fibres over the circle. He also proves that the group of the 5-twist-spun trefoil is just the direct product of the fundamental group of the fibre with the integers. We generalise this by showing that, for torus knots, the group of the twist-spun knot is such a direct product whenever the fibre is a homology sphere. This then suggests the question (asked by Zeeman for the case of the 5-twistspun trefoil) as to whether there is a corresponding product structure in the geometry. We answer in the negative.
\end{abstract}

1. Let $K \subset S^{3}$ be a knot, and $M_{k}$ its $k$-fold branched cyclic covering. Then if $K_{k} \subset S^{4}$ is the $k$-twist-spin of $K$, the main theorem of [5] states that $S^{4}-K_{k}$ is a fibre bundle over $S^{1}$, with fibre homeomorphic to $M_{k}-*$, the punctured $M_{k}$. In particular, there is an exact sequence

$$
1 \rightarrow \pi_{1}\left(M_{k}-*\right) \rightarrow \pi_{1}\left(S^{4}-K_{k}\right) \rightarrow Z \rightarrow 1
$$

which gives $\pi_{1}\left(S^{4}-K_{k}\right)$ as a semidirect product of $\pi_{1}\left(M_{k}-*\right) \cong \pi_{1}\left(M_{k}\right)$ and $Z$.

In [5], Zeeman discusses in detail the 5-twist-spun trefoil, and shows that in this case $\pi_{1}\left(S^{4}-K_{5}\right)$ is actually isomorphic to the direct product $\pi_{1}\left(M_{5}\right) \times Z$.

THEOREM 1. Let $K$ be a torus knot. Then $\pi_{1}\left(S^{4}-K_{k}\right) \cong \pi_{1}\left(M_{k}\right) \times Z$ if, and only if, $M_{k}$ is a homology 3-sphere.

Proof. The necessity of the condition that $M_{k}$ be a homology 3-sphere is easily established by noting that $\pi_{1}\left(S^{4}-K_{k}\right) \cong \pi_{1}\left(M_{k}\right) \times Z$ implies $H_{1}\left(S^{4}-K_{k}\right) \cong H_{1}\left(M_{k}\right) \oplus Z$. But $H_{1}\left(S^{4}-K_{k}\right) \cong Z$, by Alexander duality, and hence $H_{1}\left(M_{k}\right)=0$.

Now there is a well-known formula [2] which gives the order of $H_{1}\left(M_{k}\right)$ in terms of the Alexander polynomial of $K$, and it is not hard to show from this that if $K$ is the torus knot of type $m, n$, then $H_{1}\left(M_{k}\right)=0$ if, and only if, $(k, m n)=1 .^{2}$

Received by the editors February 10, 1971.

AMS 1970 subject classifications. Primary 55A25, 55A10; Secondary 57C45, 55F10.

Key words and phrases. Twist-spinning, branched cyclic covering, fibre bundle, torus knot, peripheral element.

${ }^{1}$ Partially supported by NSF grant GP-19964.

$2(a, b)$ will always denote the h.c.f. of $a$ and $b$. 
It is also well known that $\pi_{1}\left(S^{3}-K\right)$ has the presentation $\left(x, y: x^{m}=y^{n}\right)$. (Note that abelianisation takes $x \mapsto t^{n}, y \mapsto t^{m}$, where $t$ is a generator of $H_{1}\left(S^{3}-K\right) \cong Z$.)

Now let $a \in \pi_{1}\left(S^{3}-K\right)$ be a meridian element such that $a \mapsto t$ under abelianisation. Then $a$ can be expressed in terms of $x$ and $y, a=a(x, y)$, say (the precise expression will not matter), and an application of van Kampen's theorem shows that [5], [6]

$$
G_{k} \cong\left|x, y, a: x^{m}=y^{n}, a=a(x, y), a^{k} x=x a^{k}, a^{k} y=y a^{k}\right|,
$$

where we are writing $G_{k}$ for $\pi_{1}\left(S^{4}-K_{k}\right)$.

If we denote the centre of $G_{k}$ by $C\left(G_{k}\right)$, then clearly the elements $a^{k}$ and $x^{m}=y^{n}=u$, say, both lie in $C\left(G_{k}\right)$, and hence $u^{r} a^{s k} \in C\left(G_{k}\right)$ for all integers $r, s$.

Note also that if $\alpha: G_{k} \rightarrow Z$ is abelianisation, $\alpha\left(u^{r} a^{s k}\right)=t^{r m n+s k}$. Now if $(k, m n)=1$, there exist integers $r, s$ such that $r m n+s k=1$. Then $\alpha\left(u^{r} a^{s k}\right)=t$, and the splitting $\beta: Z \rightarrow G_{k}$ defined by $\beta(t)=u^{r} a^{s k}$ gives an isomorphism $G_{k} \cong \pi_{1}\left(M_{k}\right) \times Z$ as required.

2. Theorem 1 shows that if $K$ is the torus knot of type $m, n$, and $(k, m n)=1$, then

$$
\pi_{1}\left(S^{4}-K_{k}\right) \cong \pi_{1}\left(\left(M_{k}-*\right) \times S^{1}\right)
$$

A natural question (asked by Zeeman [5, Question 2] for the case of the 5-twist-spun trefoil) is therefore the following:

Is $S^{4}-K_{k} \cong\left(M_{k}-*\right) \times S^{1}$ ?

(Note that the answer is affirmative if $k=1$, for then $M_{k}$ is just $S^{3}$, and $K_{k}$ is unknotted.)

THEOREM 2. Let $K$ be a torus knot, and suppose $k>1$. Then $S^{4}-K_{k} \neq$ $\left(M_{k}-*\right) \times S^{1}$.

Proof. If $(k, m n) \neq 1$, then of course the manifolds are distinguished by their fundamental group, by Theorem 1 .

To prove the result for the interesting case $(k, m n)=1$, we proceed as follows.

Write $H_{k}$ for $\pi_{1}\left(\left(M_{k}-*\right) \times S^{1}\right)$, and let $G_{k}$ be as before, so that we have $G_{k} \cong H_{k} \cong \pi_{1}\left(M_{k}\right) \times Z$.

It is clear that if $P\left(H_{k}\right)$ denotes the set of peripheral elements of $H_{k}$ (see [3]), then $P\left(H_{k}\right)=1 \times Z$. In particular, $P\left(H_{k}\right) \subset C\left(H_{k}\right)$.

Now $S^{4}-K_{k} \cong\left(M_{k}-*\right) \times I / h$ (i.e., $\left(M_{k}-*\right) \times I$ with $\left(M_{k}-*\right) \times 0$ and $\left(M_{k}-*\right) \times 1$ identified via the homeomorphism $\left.(x, 0) \mapsto(h(x), 1)\right)$, where $h: M_{k} \rightarrow M_{k}$ is a homeomorphism such that $h(*)=*$ (in fact, $h$ is the canonical covering homeomorphism of $M_{k}$ [5]). Inclusion induces an isomorphism $G_{k}=\pi_{1}\left(\left(M_{k}-*\right) \times I / h\right) \cong \pi_{1}\left(M_{k} \times I / h\right)$; let $\gamma \in G_{k}$ be the element corresponding to that represented by $* \times I / h$. Then clearly $\gamma \in P\left(G_{k}\right)$. 
Moreover, if $\xi \in \pi_{1}\left(M_{k}-*\right) \subset \pi_{1}\left(\left(M_{k}-*\right) \times I / h\right)$, we have the formula $\gamma^{-1} \xi \gamma=h_{\#}(\xi)$. In fact, if $\left(x_{i}: r_{j}\right)$ is a presentation of $\pi_{1}\left(M_{k}-*\right), G_{k}$ has the presentation

$$
\left(x_{i}, \gamma: r_{j}, \gamma^{-1} x_{i} \gamma=h_{\#}\left(x_{i}\right)\right) .
$$

In particular, if $\gamma \in C\left(G_{k}\right)$, we would have $h_{\#}=$ the identity.

But sewing back the knot $K_{k}$ (to get $S^{4}$ ) has the effect of adding the relation $\gamma=1$ to $G_{k}$, and therefore $\left|x_{i}: r_{j}, x_{i}=h_{\#}\left(x_{i}\right)\right|$ has to be the trivial group. If $h_{\#}=$ the identity, however, this group is just $\pi_{1}\left(M_{k}\right)$, so if we show that $\pi_{1}\left(M_{k}\right) \neq 1$, we are through. For we will then have proved that there exists an element of $P\left(G_{k}\right)$ (namely $\gamma$ ) which does not lie in $C\left(G_{k}\right)$, whereas $P\left(H_{k}\right) \subset C\left(H_{k}\right)$. Hence $\left(M_{k}-*\right) \times I / h$ and $\left(M_{k}-*\right) \times S^{1}$ cannot be homeomorphic.

LEMmA. If $M_{k}$ is the $k$-fold branched cyclic covering of a torus knot, and $k>1$, then $\pi_{1}\left(M_{k}\right) \neq 1$.

Proof. Recalling the exact sequence in $\S 1$, it will be sufficient to prove that $G_{k} \neq \boldsymbol{Z}$.

Let $K$ be the torus knot of type $m, n$. Its group $\pi_{1}\left(S^{3}-K\right) \cong\left|x, y: x^{m}=y^{n}\right|$, and if $r, s$ are integers such that $r m+s n=1$ (such integers exist since $(m, n)=1)$, then $y^{r} x^{s}$ is a meridian element (see for example [4]). It follows that $G_{k}$ has the presentation

$$
\left(x, y: x^{m}=y^{n},\left(y^{r} x^{s}\right)^{k} x=x\left(y^{r} x^{s}\right)^{k},\left(y^{r} x^{s}\right)^{k} y=y\left(y^{r} x^{s}\right)^{k}\right),
$$

and hence maps homomorphically onto the group

$$
\left|x, y: x^{m}=y^{n}=\left(y^{r} x^{s}\right)^{k}=1\right| .
$$

Sending $x \mapsto u^{n}$ and $y \mapsto v^{m}$ then defines a homomorphism from $\left|x, y: x^{m}=y^{n}=\left(y^{r} x^{s}\right)^{k}=1\right|$ onto $\left|u, v: u^{m}=v^{n}=(v u)^{k}=1\right|$. (The map is a homomorphism since the relations $u^{n m}=1, v^{m n}=1,\left(v^{m r} u^{n s}\right)^{k}=1$ are all consequences of the relations $u^{m}=1, v^{n}=1,(v u)^{k}=1$, and it is onto since $u=u^{n s}, v=v^{m r}$.)

But this latter group is just the polyhedral group $(m, n, k)$ (see [1]), which is nonabelian unless $m=1, n=1$, or $k=1$, or $m=n=k=2$, cases which are not relevant here. Hence $G_{k}$ is nonabelian.

\section{REFERENCES}

1. H. S. M. Coxeter and W. O. J. Moser, Generators and relations for discrete groups, Springer-Verlag, Berlin, 1957. MR 19, 527.

2. R. H. Fox, Free differential calculus. III: Subgroups, Ann. of Math. (2) 64 (1956), 407-419. MR 20 \#2374. 
3. - On the complementary domains of a certain pair of inequivalent knots, Nederl. Akad. Wetensch. Proc. Ser. A 55=Indag. Math. 14 (1952), 37-40. MR 13, 966.

4. J. Hempel, $A$ simply connected 3-manifold is $S^{3}$ if it is the sum of a solid torus and the complement of a torus knot, Proc. Amer. Math. Soc. 15 (1964), 154-158. MR 28 \#599.

5. E. C. Zeeman, Twisting spun knots, Trans. Amer. Math. Soc. 115 (1965), 471-495. MR 33 \#3290.

6. R. H. Fox, Some n-dimensional manifolds that have the same fundamental group, Michigan Math. J. 15 (1968), 187-189.

Department of Mathematics, Florida State University, Tallahassee, Florida 32306 